\title{
THE BIG DEHN SURGERY GRAPH AND THE LINK OF $S^{3}$
}

\author{
NEIL R. HOFFMAN AND GENEVIEVE S. WALSH
}

(Communicated by Martin Scharlemann)

Dedicated to Bill Thurston

\begin{abstract}
In a talk at the Cornell Topology Festival in 2004, W. Thurston discussed a graph which we call "The Big Dehn Surgery Graph", B. Here we explore this graph, particularly the link of $S^{3}$, and prove facts about the geometry and topology of $\mathcal{B}$. We also investigate some interesting subgraphs and pose what we believe are important questions about $\mathcal{B}$.
\end{abstract}

\section{INTRODUCTION}

In unpublished work, $\mathrm{W}$. Thurston described a graph that had a vertex $v_{M}$ for each closed, orientable, 3-manifold $M$ and an edge between two distinct vertices $v_{M}$ and $v_{M^{\prime}}$, if there exists a Dehn surgery between $M$ and $M^{\prime}$. That is, there is a knot $K \subset M$ and $M^{\prime}$ is obtained by non-trivial Dehn surgery along $K$ in $M$. The edges are unoriented since $M$ is also obtained from $M^{\prime}$ via Dehn surgery. Roughly following W. Thurston, we will call this graph the Big Dehn Surgery Graph, denoted by $\mathcal{B}$. We will sometimes denote the vertex $v_{M}$ by $M$. If $M$ and $M^{\prime}$ are obtained from one another via Dehn surgery along two distinct knots, we do not make two distinct edges, although this would also make an interesting graph. We first record some basic properties of $\mathcal{B}$. These follow from just some of the extensive work that has been done in the field of Dehn surgery.

Proposition 1.1. The graph $\mathcal{B}$ has the following basic properties: (i) $\mathcal{B}$ is connected; (ii) $\mathcal{B}$ has infinite valence; and (iii) $\mathcal{B}$ has infinite diameter.

The graph $\mathcal{B}$ is connected by the beautiful work of Lickorish [21] and Wallace 35 . who independently showed that all closed, orientable 3 -manifolds can be obtained by surgery along a link in $S^{3}$. That every vertex $v_{M}$ in $\mathcal{B}$ has infinite valence can be seen, amongst other ways, by constructing a hyperbolic knot $K$ in $M$ via the work of Myers in 26. Then by work of Thurston 33 all but finitely many fillings are hyperbolic, and the volumes of the filled manifolds approach the volume of the cusped manifold. The graph $\mathcal{B}$ has infinite diameter since the rank of $H_{1}(M, \mathbb{R})$ can change by at most one via drilling and filling, and there are 3-manifolds with arbitrarily high rank.

The Lickorish proof explicitly constructs a link, and therefore allows us to describe a natural notion of distance. A shortest path from $v_{S^{3}}$ to $v_{M}$ in $\mathcal{B}$ counts the minimum number of components needed for a link in $S^{3}$ to admit $M$ as a surgery. We will refer to the number of edges in a shortest edge path between $v_{M_{1}}$ and $v_{M_{2}}$

Received by the editors March 25, 2015 and, in revised form, June 5, 2015 and July 29, 2015. 2010 Mathematics Subject Classification. Primary 57M25; Secondary 57M50. 
as the Lickorish path length and denote this function by $p_{L}\left(v_{M_{1}}, v_{M_{2}}\right)$. For example, if $P^{3}$ denotes the Poincaré homology sphere, then $p_{L}\left(S^{3}, P^{3} \# P^{3}\right)=2$. See $\$ 3$ for more on $p_{L}$. Lickorish path length appears in the literature as surgery distance (see [3], 18]). This gives us a metric on $\mathcal{B}$, which we assume throughout the paper.

The Big Dehn Surgery Graph is very big. In order to get a handle on it, we will study some useful subgraphs. We denote the subgraph of a graph generated by the vertices $\left\{v_{i}\right\}$ by $\left\langle\left\{v_{i}\right\}\right\rangle$. The link of a vertex $v$ is the subgraph $l k(v)=\langle w$ : $\left.p_{L}(v, w)=1\right\rangle$. If there is an automorphism of $\mathcal{B}$ taking a vertex $v$ to a vertex $w$, then the links of $v$ and $w$ are isomorphic as graphs. We study the links of vertices and a possible characterization of the link of $S^{3}$ in $\$ 2$. Associated to any knot $K$ in a manifold $M$ is a $\mathbb{K}_{\infty}$, the complete graph on infinitely many vertices (to distinguish between knots and complete graphs we will use $\mathbb{K}_{\infty}$ and $\mathbb{K}_{n}$ to denote complete graphs). When we want to describe a $\mathbb{K}_{\infty}$ subgraph of $\mathcal{B}$, we will use $M_{\infty}^{K}=\left\langle v_{M^{\prime}}: M^{\prime}=M(K ; r)\right\rangle$. See $\oint 2$ for notation conventions used in this paper.

Interestingly, not every $\mathbb{K}_{\infty}$ arises this way. We prove this in $\oiint_{4}$ and make some further observations about these subgraphs. In $₫ 6$ we study the subgraph $\mathcal{B}_{H}$. The vertices of the subgraph $\mathcal{B}_{H}$ are closed hyperbolic 3 -manifolds and there is an edge between two vertices $v_{M}$ and $v_{N}$ if there is a cusped hyperbolic 3-manifold with two fillings homeomorphic to $M$ and $N$. We also study the geometry of $\mathcal{B}$ and $\mathcal{B}_{H}$, showing that neither is $\delta$-hyperbolic in $\$ 7$ In $\$ 7$ we also construct flats of arbitrarily large dimension in $\mathcal{B}$. An infinite family of hyperbolic 3-manifolds with weight one fundamental group which are not obtained via surgery on a knot in $S^{3}$ is given in 93 . This shows that a characterization of the vertices in the link of $S^{3}$ remains open. Bounded subgraphs whose vertices correspond to other geometries are detailed in $\$ 5$

\section{THE LINK OF $S^{3}$}

We now set notation which we will use for the remainder of the paper. A slope on the boundary of a 3-manifold $M$ is an isotopy class of unoriented, simple closed curves on $\partial M$. We denote the result of Dehn surgery on $M$ along a knot $K \subset$ $M$ with filling slope $r$ by $M(K ; r)$. We denote Dehn filling along a link $L=$ $K_{1} \cup K_{2} \cdots \cup K_{n} \subset M$ by $M\left(\left\{K_{1}, \ldots, K_{n}\right\} ;\left(r_{1}, \ldots, r_{n}\right)\right)$ or $M\left(L ;\left(r_{1}, \ldots, r_{n}\right)\right)$, with a dash denoting an unfilled component. Thus the exterior of $K$ in $M$ is denoted $M(K ;-)$ and the complement is denoted by $M \backslash K$. We will say that $M(K ;-)$ or $M \backslash K$ is hyperbolic if its interior admits a complete hyperbolic metric of finite volume. For knot and link exteriors in $S^{3}$ we will frame the boundary tori homologically, unless otherwise noted.

Here we study the links of vertices in $\mathcal{B}$, particularly the link of $S^{3}$. As above, the link of a vertex in $\mathcal{B}$ is the subgraph $l k(v)=\left\langle w: p_{L}(v, w)=1\right\rangle$. If $v$ is associated to the manifold $M$, the vertices in this subgraph correspond to distinct manifolds which can be obtained via Dehn surgery on knots in $M$. We refer to this subgraph as the link of $M$ in $\mathcal{B}$, or just the link of $M$.

The link of $S^{3}$ in $\mathcal{B}$ is connected. There are several proofs of this fact. Perhaps the most intuitive is to use that a crossing change on a knot in $S^{3}$ can be realized as a Dehn surgery along an unknotted circle; see [31. One must be careful to ensure that none of these surgeries results in $S^{3}$.

The proof we give here arose from conversations with Luisa Pauoluzzi, and the path shows that the link of $S^{3}$ has bounded diameter. 
Proposition 2.1. The link of $S^{3}$ in $\mathcal{B}$ is connected and of bounded diameter.

Proof. We show any surgery on a knot in $S^{3}, S^{3}(K ; r)$, is at most distance three in the link from a lens space. Let $C K$ denote a cable of $K$. Then there is a surgery slope $p q$ and a lens space $L(p, q)$ such that $S^{3}(C K ; p q)=S^{3}(K ; p / q) \# L(p, q)$; see [10]. Thus $S^{3}(C K ; p q)$ is distance one from a surgery along $K$ and distance one from a lens space.

One might hope to distinguish the links of vertices combinatorially in $\mathcal{B}$. For example, is the link of any vertex in $\mathcal{B}$ connected? of bounded diameter? A negative answer would lead to an obstruction to automorphisms of the graph that do not fix $S^{3}$. More generally, an answer to the following question would lead to a better understanding of how the Dehn surgery structure of a manifold relates to the homeomorphism type.

Question 2.2. Does the graph $\mathcal{B}$ admit a non-trivial automorphism?

Given our results below in 93 , we do not know of a conjectured answer to the following problem, which amounts to characterizing manifolds obtained via surgery on a knot in $S^{3}$.

Problem 2.3. Characterize the vertices in the link of $S^{3}$.

\section{Hyperbolic EXAMPles With WEIGHT ONE FUndAMENTAL GROUPS}

A group is weight $n$ if it can be normally generated by $n$ elements and no normal generating set with fewer elements exists. Recall that all knot groups are weight one and hence all manifolds obtained by surgery along a knot in $S^{3}$ have weight one fundamental groups. It is a folklore question if a manifold which admits a geometric structure and has a weight one fundamental group can always be realized as surgery along a knot in $S^{3}$ (see [1, Question 9.23]). The restriction to geometric manifolds is necessary since the fundamental group of $P^{3} \# P^{3}$ is weight one, where $P^{3}$ is the Poincaré homology sphere. This cannot be surgery along a knot in $S^{3}$ since if a reducible manifold is surgery along a non-trivial knot in $S^{3}$, one of the factors is a lens space 11 .

In Theorem 3.4 we show that there are infinitely many hyperbolic 3 -manifolds whose fundamental groups are normally generated by one element but which are not in the link of $S^{3}$ (Theorem 3.4). Our technique is a generalization to the hyperbolic setting of a method of Margaret Doig, who in 8 first came up with examples that could not be obtained via surgery on a knot in $S^{3}$ using the $d$-invariant. Boyer and Lines [5] exhibited a different set of small Seifert fibered spaces which are weight one but not surgery along a knot in $S^{3}$.

Before describing the hyperbolic examples, we make a few remarks regarding the weight one condition. We have the following obstruction to surgery due to James Howie:

Theorem 3.1 ([17, Corollary 4.2]). Every one relator product of three cyclic groups is non-trivial.

This implies, for example, that $M \cong L\left(p_{1}, q_{1}\right) \# L\left(p_{2}, q_{2}\right) \# L\left(p_{3}, q_{3}\right)$, is not obtained via surgery on a knot in $S^{3}$, since its fundamental group is not weight one. However, when the $p_{i}$ are pairwise relatively prime, its homology is cyclic.

The following proposition extends this consequence of Howie's result to hyperbolic manifolds. 


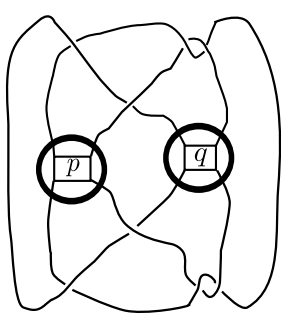

(a)

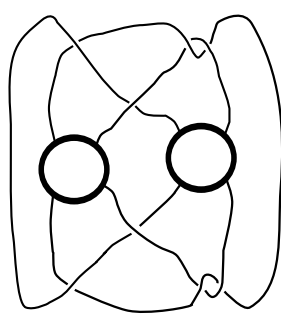

(b)

Figure 1. The The Kanenobu knots $K_{p, q}$ (left) and the tangle obtained by drilling the $p$ and $q$ twist regions (right).

Proposition 3.2. There are hyperbolic 3-manifolds $\left\{N_{j}\right\}$ with cyclic homology such that for each $j, \pi_{1}\left(N_{j}\right)$ is weight at least two.

Proof. Just as above, $M \cong L\left(p_{1}, q_{1}\right) \# L\left(p_{2}, q_{2}\right) \# L\left(p_{3}, q_{3}\right)$ with all the $p_{i}$ pairwise relatively prime. By [26, Theorem 1.1], there exists a knot $K \subset M$ such that $K$ bounds an immersed disk in $M$ and $M-K$ is hyperbolic. Denote by $\Gamma_{K}=$ $\pi_{1}(M(K,-))$ and $\pi_{1}(\partial(M(K ;-)))=\langle\mu, \lambda \mid[\mu, \lambda]=1\rangle$, where $\mu$ and $\lambda$ are chosen such that $M(K ; \mu)=M$, and $\lambda$ bounds an immersed disk when considered as a curve in $M$.

Let $\langle\langle g\rangle\rangle_{G}$ denote the normal closure of $g$ in $G$. If $\gamma$ is a curve in $\partial(M(K ;-))$ representing the isotopy class $\mu^{r} \lambda^{s}$, then $\pi_{1}(M(K ; \gamma))=\Gamma_{K} /\left\langle\left\langle\mu^{r} \lambda^{s}\right\rangle\right\rangle_{\Gamma_{K}}$. Observe that $\Gamma_{K} /\left\langle\left\langle\mu^{r} \lambda^{s}, \mu\right\rangle\right\rangle_{\Gamma_{K}}=\Gamma_{K} /\langle\langle\mu\rangle\rangle_{\Gamma_{K}}=\pi_{1}(M)$ as $\lambda \in\langle\langle\mu\rangle\rangle_{\Gamma_{K}}$ since $\lambda$ bounds an immersed disk in $M$. Thus, there exists a surjective homomorphism $f: \pi_{1}(M(K ; \gamma)) \rightarrow \pi_{1}(M)$. In particular, $\pi_{1}(M(K ; \gamma))$ is weight at least two.

If we let $N_{j}=M\left(K ; \mu \lambda^{j}\right)$, then $H_{1}\left(N_{j}, Z\right)$ is cyclic of order $p_{1} p_{2} p_{3}$ and by Thurston's Hyperbolic Dehn Surgery Theorem [33. Theorem 5.8.2], $N_{j}$ is hyperbolic for sufficiently large $j$.

Over the two papers 2, 3, Dave Auckly exhibited hyperbolic integral homology spheres that could not be surgery along a knot in $S^{3}$. However, it is unknown if these examples have weight one since his construction involves a 4-dimensional cobordism that preserves homology, but not necessarily group weight.

Margaret Doig has recently exhibited examples of manifolds admitting a Thurston geometry, but which cannot be obtained by surgery along a knot in $S^{3}$. As part of a larger result, she shows:

Theorem 3.3 ([8, Theorem 2]). Of the infinite family of elliptic manifolds with $H_{1}(Y)=\mathbb{Z} / 4 \mathbb{Z}$ and non-cyclic fundamental group, only one (up to homeomorphism) can be realized as surgery on a knot in $S^{3}$, and that is $(4,1)$ surgery on the right-handed trefoil.

Although not explicitly stated in her result, for a finite group $G$, the weight of $G$ is determined by the weight $G / G^{\prime}$ (see [20]), and so the above elliptic manifolds have weight one fundamental groups.

Using similar techniques and the work of Greene and Watson in 13, we are able to exhibit hyperbolic manifolds that have weight one fundamental groups but are never surgery along a knot in $S^{3}$. As in Greene and Watson, our examples are 
the double branched covers of the knots $K_{p, q}$ (see Figure 1(a) where $p=-10 n$, $q=10 n+3$, and $n \geq 1$. We denote these knots by $K_{n}$ and their corresponding double branched covers by $M_{n}$. The techniques of the proof may require us to omit finitely many of these double branched covers from the statement of the theorem. We will use $\left\{X_{n}\right\}$ to denote the manifolds in this (possibly) pared down set.

Theorem 3.4. There is an infinite family of hyperbolic manifolds, $\left\{X_{n}\right\}$, none of which can be realized as surgery on a knot in $S^{3}$. Furthermore, these manifolds have weight one fundamental groups.

In the following proof, we require two standard definitions from Heegaard-Floer homology (see [28, 8]). First, a rational homology sphere $M$ is an $L$-space if the hat version of its Heegaard-Floer homology is as simple as possible, namely for each $\operatorname{Spin}^{c}$ structure $t$ of $M$, the hat version of $\widehat{H F}(M, t)$ has a single generator and no cancellation. The $d$-invariant, $d(M, t)$, is an invariant assigned to each Spin $^{c}$ structure $t$ of $M$ which records the minimal degree of any non-torsion class of $H F^{+}(M, t)$ coming from $H F^{\infty}(M, t)$; see section 4 of [28]. Crudely, the $d$-invariant can be thought of as a way of measuring how far from $S^{3}$ a manifold is. This mentality is motivated by the argument in the proof below.

Proof. For this proof, we use notation from 30. As noted above, Greene and Watson [13] study the family of knots $\left\{K_{n}\right\}$ and their double branched covers $M_{n}$. The manifolds $M_{n}$ have the following properties:

Each $M_{n}$ is an $L$-space ([13, Proposition 11]).

The $d$-invariant, defined in 28, of the $M_{n}$, satisfies the following relation:

$$
d\left(M_{n}, i\right)=2 \tau\left(M_{n}, i\right)-\lambda
$$

for all $n \geq 0$ and all $i \in \operatorname{Spin}^{c}\left(M_{n}\right)$. Here $\tau\left(M_{n}, i\right)$ is the Turaev torsion and $\lambda=\lambda\left(M_{n}\right)$ is the Casson-Walker invariant. That the Casson-Walker invariants are all identical follows from the work of Mullins [25, Theorem 7.1] and that the knots are ribbon and have identical Jones polynomials [13, Propositions 8 and 11]. Furthermore, by [13, Proposition 14],

$$
\lim _{n \rightarrow \infty} \min \left\{\tau\left(M_{n}, i\right) \mid i \in \operatorname{Spin}^{c}\left(M_{n}\right)\right\}=-\infty .
$$

As they observe, (1) and (2) above imply:

$$
\lim _{n \rightarrow \infty} \min \left\{d\left(M_{n}, i\right) \mid i \in \operatorname{Spin}^{c}\left(M_{n}\right)\right\}=-\infty .
$$

Let $-M_{n}$ denote $M_{n}$ with the opposite orientation. Then since $d\left(-M_{n}, i\right)=$ $-d\left(M_{n}, i\right)$, 28, Proposition 4.2], we have:

$$
\lim _{n \rightarrow \infty} \max \left\{d\left(-M_{n}, i\right) \mid i \in \operatorname{Spin}^{c}\left(M_{n}\right)\right\}=\infty .
$$

In particular, the $d$-invariants are unbounded with either orientation.

Since the manifolds $M_{n}$ (and $-M_{n}$ ) are $L$-spaces, we may apply:

Theorem 3.5 ([28, Theorem 1.2]). If a knot $K \subset S^{3}$ admits an L-space surgery, then the non-zero coefficients of $\Delta_{K}(T)$ are alternating $+1 s$ and $-1 s$.

For the remainder of this section, we will consider $S_{p / q}^{3}(K)$ with $p / q \neq 1 / 0$. We assume that $p / q \geq 0$ by the following argument. Let $m K$ denote the mirror of $K$. Then, $S_{p / q}^{3}(K)=S_{-p / q}^{3}(m K)$ and we replace $K$ with $m K$ if necessary to fulfill the assumption that $p / q \geq 0$. The resulting manifold will be $S_{p / q}^{3}(K)$ with the 
opposite orientation. Note that a knot which admits an $L$-space surgery cannot be amphichiral [8, Corollary 12].

If a knot surgery $S_{p / q}^{3}(K)$ with $p / q \geq 0$ is an $L$-space, it is shown in [30, Theorem 1.2] that the correction terms $d\left(S_{p / q}^{3}(K), i\right)$ may be calculated as follows, for $|i| \leq$ $p / 2$, and $c=|\lfloor i / q\rfloor|$ :

$$
d\left(S_{p / q}^{3}(K), i\right)-d\left(S_{p / q}^{3}(U), i\right)=-2 \sum_{j=1}^{\infty} j a_{c+j}
$$

where $a_{i}$ is defined in terms of the normalized Alexander polynomial of $K$ :

$$
\Delta_{K}(T)=a_{0}+\sum_{i=1}^{n} a_{i}\left(T^{i}+T^{-1}\right) .
$$

Again, we are using notation from [30] and in particular $S_{p / q}^{3}(K)=S^{3}(K ; p / q)$. We note that Greene and Watson [13] establish that $H_{1}\left(M_{n}\right)=\mathbb{Z} / 25 \mathbb{Z}$. By homology considerations, if any $M_{n}$ is $p / q$ surgery on a (standard positively framed) knot $K$ in $S^{3}$, then $p=25$. The $L$-space condition implies $\frac{25}{q} \geq 2 g(K)-1$ by [30, Corollary 1.4]. We also know that such a $K$ is fibered by [19, 27] and that $g(K)$ is the degree of the symmetrized Alexander polynomial of $K$ by [29], bounding the number of terms on the right-hand side of equation (5).

Now suppose that $M_{n}=S_{p / q}^{3}(K)$. Then, since $M_{n}$ and $-M_{n}$ are $L$-spaces, the Alexander polynomials of such a $K$ have bounded coefficients by Theorem 3.5 . Thus, the right-hand side of equation (5) is bounded. Since there are only finitely many $L(p, q)$ with $p=25, d\left(S_{p / q}^{3}(U), i\right)$ only can take on finitely many values. Therefore, $d\left(S_{p / q}^{3}(K), i\right)$ is globally bounded whenever $S_{p / q}^{3}(K)=M_{n}$. However, this contradicts the limit (3) or 40), and so at most finitely many of the $M_{n}$ can be surgery on any knot.

Next, we establish that all but at most finitely many $\left\{M_{n}\right\}$ are hyperbolic.

Indeed, the Kanenobu knots $K_{n}$ are all obtained by tangle filling the two boundary components of the tangle $T$ in Figure 1(b) and so the manifolds $\left\{M_{n}\right\}$ are obtained by Dehn filling the double cover of $T$, which we denote by $M$. A triangulation for $M$ can be obtained by inputting $T$ labeled with cone angle $\frac{\pi}{2}$ into the computer software Orb (an orbifold version of the original Snappea) [16] to obtain an orbifold structure $Q 11$ Denote by $M$, the double cover of $Q$ corresponding to the unique index 2 torsion free subgroup $\pi_{1}^{\text {orb }}(Q)$. This computation shows that $M$ decomposes into 8 tetrahedra. In fact, SnapPy's identify function [6] shows $M$ is homeomorphic to ' $\mathrm{t} 12060$ ' in the 8 tetrahedral census. Also, using SnapPy, a set of 8 gluing equations for $M$ are encoded by the following matrix:

$$
\left(\begin{array}{rrrrrrrrrrrrrrrr|r}
2 & -2 & 2 & 1 & 2 & -1 & 1 & -1 & 2 & 0 & 0 & 2 & -1 & 1 & 2 & 0 & -4 \\
0 & 0 & 0 & 0 & 0 & 0 & 1 & -1 & 0 & 0 & 0 & 0 & -1 & 1 & 0 & 0 & 0 \\
-1 & 2 & 0 & -1 & 0 & -1 & 0 & 0 & -2 & 1 & 0 & 0 & 0 & 0 & 0 & 0 & 2 \\
0 & -2 & -1 & 1 & -1 & 1 & 0 & -1 & 0 & 0 & 0 & 0 & 0 & -1 & 0 & 0 & 0 \\
1 & 0 & 1 & 0 & 1 & 0 & 0 & 0 & 0 & 0 & 1 & 0 & 0 & 0 & 1 & 0 & -2 \\
0 & 0 & 1 & 0 & 1 & 0 & 1 & 0 & 2 & 0 & 0 & 0 & 1 & 0 & 0 & 0 & -2 \\
0 & 0 & 0 & -1 & 0 & -1 & -1 & 1 & 0 & 0 & -1 & 1 & -1 & 1 & -1 & 1 & 2 \\
0 & 0 & 0 & 0 & 0 & 0 & -1 & 1 & 0 & 0 & 1 & -1 & 1 & -1 & -1 & 1 & 0
\end{array}\right) .
$$

\footnotetext{
${ }^{1}$ The file and instructions on how to use it are available on the arXiv version of this paper.
} 


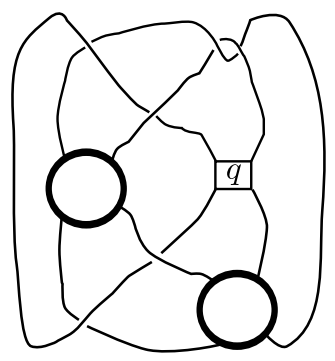

(a) We drill out two crossing regions.

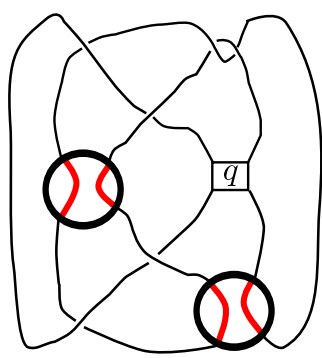

(b) Then we fill as above.

Figure 2. These diagrams show $K_{n}$ switching two tangle regions produces the unknot

The coding is as follows: given a row $\left(\begin{array}{llllllll}a_{1} & b_{1} & a_{2} & b_{2} & \ldots & a_{8} & b_{8} & \mid c\end{array}\right)$, we produce a $\log$ equation $a_{1} \log \left(z_{1}\right)+b_{1} \log \left(1-z_{1}\right)+\cdots+a_{8} \log \left(z_{8}\right)+b_{8} \log \left(1-z_{8}\right)-c \pi$. $i=0$. Given such an encoding $z=\left(2 i, \frac{1}{5}+\frac{3 i}{5}, \frac{1}{5}+\frac{3 i}{5}, \frac{1}{2}+\frac{i}{2}, 1+2 i, \frac{1}{2}+i, \frac{1}{2}+\frac{i}{2}, \frac{1}{2}+i\right)$ is an exact solution and therefore $M$ and 't12060' admit a complete hyperbolic structure. By Thurston's Hyperbolic Dehn Surgery Theorem [33. Theorem 5.8.2], the manifolds $M_{n}$ limit to $M$. Thus, there are at most finitely many non-hyperbolic $M_{n}$.

We have that at most finitely many of the $M_{n}$ are surgery on a knot and at most finitely many are non-hyperbolic. We denote the subsequence of $M_{n}$ that are hyperbolic and cannot be surgery along a knot by $X_{n}$.

Finally, we establish that $\pi_{1}\left(M_{n}\right)$ is weight one and therefore $\pi_{1}\left(X_{n}\right)$ is weight one. As noted in [13, §4.2],

$$
\begin{aligned}
\pi_{1}\left(M_{n}\right)=\left\langle a_{1}, a_{2}, a_{3}, a_{4} \mid b_{1}, b_{2}, b_{3}, b_{4}\right\rangle \text { with } & b_{1}=\left(a_{1}^{-1} a_{2}\right)^{10 n} a_{4}^{-1} a_{1}^{2}, \\
& b_{2}=a_{2}^{-1} a_{3}\left(a_{2}^{-1} a_{1}\right)^{10 n} a_{2}^{-1}, \\
& b_{3}=\left(a_{4}^{-1} a_{3}\right)^{10 n+3} a_{3}^{-1} a_{2} a_{3}^{-2}, \text { and } \\
& b_{4}=a_{1}^{-1} a_{4}\left(a_{3}^{-1} a_{4}\right)^{10 n+3} a_{4}^{2} .
\end{aligned}
$$

We claim $\pi_{1}\left(M_{n}\right) /\left\langle\left\langle a_{1}\right\rangle\right\rangle_{\pi_{1}\left(M_{n}\right)}$ is trivial. First, the relations $b_{1}, b_{2}$ become $a_{2}^{10 n}=a_{4}$ and $a_{2}^{10 n+2}=a_{3}$, respectively. Also, the relations $b_{3}$ and $b_{4}$ reduce to $a_{2}^{10 n-1}=1$ and $a_{2}^{10 n-6}=1$ respectively. The claim follows as $\operatorname{gcd}(10 n-1,10 n-6)=$ 1 .

Corollary 3.6. For all $n, p_{L}\left(M_{n}, S^{3}\right) \leq 2$ and for all but at most finitely many $n$, $p_{L}\left(M_{n}, S^{3}\right)=2$.

Proof. Since we can produce the unknot by switching two crossing regions of the diagram for $K_{n}$ as in Figure 2, the Montesinos trick shows that $M_{n}$ can be obtained from surgery along a two component link in $S^{3}$. Hence, we see the upper bound $p_{L}\left(M_{n}, S^{3}\right) \leq 2$ and $p_{L}\left(M_{n}, S^{3}\right) \geq 2$ is established for all but at most finitely many $n$ by Theorem 3.4 .

Remark 3.7. In 22, Marengon extends the techniques given here to exhibit an infinite four parameter family of double branched covers of knots given by a Kaneobu like construction. 


\section{Complete infinite SUbGraphs}

Here we discuss an interesting property which may allow one to "see" knots in the graph $\mathcal{B}$. We also want to employ the notion of the set of neighbors of a vertex in a graph. More formally, for a graph $G$ and a subset $\left\{w_{i}\right\}$ of the vertices of $G$, let $\left\langle\left\{w_{i}\right\}\right\rangle$ be the subgraph induced by these vertices. That is, the vertices of $\left\langle\left\{w_{i}\right\}\right\rangle$ are $\left\{w_{i}\right\}$, and $\left(w_{i}, w_{j}\right)$ is an edge of $\left\langle\left\{w_{i}\right\}\right\rangle$ exactly when $\left(w_{i}, w_{j}\right)$ is an edge of $G$. Then, as in the introduction, we define the link a vertex $v$ in $G$ to be $\left\langle\left\{w_{i}\right\}\right\rangle$, for all $w_{i}$ which are path length one from $v$.

Definition 4.1. If $K$ is a knot in a 3-manifold $M$, then $(M)_{\infty}^{K}=\left\langle\left\{v_{M(K ; r)}\right\}\right\rangle$, where $\{M(K ; r)\}$ is the set of 3 -manifolds obtained from $M$ via Dehn surgery on $K$.

Proposition 4.2. For any closed 3-manifold $M$ and knot $K \subset M,(M)_{\infty}^{K}$ is a $\mathbb{K}_{\infty}$.

Proof. That every vertex in $M_{\infty}^{K}$ is connected to every other one is a consequence of the definition. We just need to observe that there are infinitely many different manifolds in this subgraph. If $M \backslash K$ admits a hyperbolic structure, then all but finitely many fillings are hyperbolic. Furthermore, the volumes approach the volume of $M \backslash K$ and so there are infinitely many different homeomorphism types. If $M \backslash K$ is Seifert-fibered (including the unknot complement in $S^{3}$ ), it is Seifert-fibered over an orbifold $O$ with boundary. The fillings $r$ can be chosen so that the result is Seifert-fibered over an orbifold where the boundary component of $O$ is replaced with a cone point of arbitrarily high order, so the Seifert-fibered spaces are not homeomorphic. If $M \backslash K$ admits a decomposition along incompressible tori, then infinitely many fillings have this same decomposition [12]. Then the boundary of $M \backslash K$ is in either a hyperbolic piece or a Seifert-fibered piece, and the above arguments apply. Finally, if $M \backslash K$ is reducible, then there exists a separating $S^{2}$ such that $M \backslash K=M_{1} \# M_{2} \backslash K$ where $M_{2} \backslash K$ is irreducible. In this case, the previous arguments can be applied to yield the desired result.

Note that, sometimes, the intersection of two $\mathbb{K}_{\infty}$ subgraphs arising from fillings on knot complements may intersect in a $\mathbb{K}_{\infty}$. For example, let $U$ be the unknot and $T_{r, s}$ a torus knot. Let $\left(S^{3}\right)_{\infty}^{U}$ be the $\mathbb{K}_{\infty}$ associated to $S^{3} \backslash U$, and $\left(S^{3}\right)_{\infty}^{T_{r, s}}$ be the $\mathbb{K}_{\infty}$ associated to $S^{3} \backslash T_{r, s}$. Then $\left(S^{3}\right)_{\infty}^{U} \cap\left(S^{3}\right)_{\infty}^{T_{r, s}}$ is a $\mathbb{K}_{\infty}$ where each vertex is a lens space (see [24]). However, this phenomena cannot happen for hyperbolic manifolds.

Proposition 4.3. If $M \backslash K$ and $M^{\prime} \backslash K^{\prime}$ are hyperbolic and not homeomorphic, then the subgraphs $(M)_{\infty}^{K}$ and $\left(M^{\prime}\right)_{\infty}^{K^{\prime}}$ have at most finitely many vertices in common.

Proof. Assume that $(M)_{\infty}^{K}$ and $\left(M^{\prime}\right)_{\infty}^{K^{\prime}}$ have infinitely many vertices in common. Then infinitely many of these are hyperbolic. Denote this set by $\left\{N_{i}\right\}_{i} \in \mathbb{N}$. Choose a basepoint in the thick part of each $N_{i}$. Then the geometric limit of the $N_{i}$ is $M \backslash K$ and it is also $M^{\prime} \backslash K^{\prime}$, so they must be homeomorphic. See 14] for background on geometric limits.

\subsection{Subgraphs which do not arise from filling.}

Theorem 4.4. There is a $\mathbb{K}_{\infty}$ of small Seifert-fibered spaces that does not come from surgery along a one cusped manifold. 
Proof. We will construct a family $M_{i, j}, i \in\{1,2,3,4\}, j \in \mathbb{N}$, of Seifert-fibered spaces over an orbifold with base space $S^{2}$ and negative Euler characteristic. We follow notation in [15. In particular, we denote a closed Seifert-fibered space by $S F S\left(F ; \alpha_{1} / \beta_{1}, \ldots, \alpha_{n} / \beta_{n}\right)$ where $F$ is the underlying space of the base orbifold. The cone points of the base orbifold will have multiplicities $\beta_{i}$. The Seifert-fibered invariants of the exceptional fibers are $\alpha_{i} / \beta_{i}$, which are allowed to take values in $\mathbb{Q}$. Two Seifert fiberings $S F S\left(F ; \alpha_{1} / \beta_{1}, \ldots, \alpha_{n} / \beta_{n}\right)$ and $S F S\left(F^{\prime} ; \alpha_{1}^{\prime} / \beta_{1}^{\prime}, \ldots, \alpha_{m}^{\prime} / \beta_{m}^{\prime}\right)$ are isomorphic by a fiber preserving diffeomorphism if and only if after possibly permuting indices, $\alpha_{i} / \beta_{i} \equiv \alpha_{i}^{\prime} / \beta_{i}^{\prime} \bmod 1$ and, if $F$ is closed, $\sum \alpha_{i} / \beta_{i}=\sum \alpha_{i}^{\prime} / \beta_{i}^{\prime}$ [15, Proposition 2.1].

Now let $\left\{a_{1} / b_{1}, a_{2} / b_{2}, a_{3} / b_{3}, a_{4} / b_{4}\right\}$ be four distinct rational numbers, such that $0<a_{i} / b_{i}<1, \sum 1 / b_{i}<1$ and $a_{i}, b_{i}$ are relatively prime. Let $M_{4,0}$ be the Seifertfibered space over $S^{2}$ with three exceptional fibers labeled by $a_{i} / b_{i}(i \neq 4)$. We can define $M_{1,0}, M_{2,0}, M_{3,0}$ similarly. The condition $\sum 1 / b_{i}<1$ ensures that each manifold will be Seifert-fibered over a hyperbolic orbifold.

Note that each manifold $M_{i, 0}$ has exactly two common exceptional fibers with the others mod 1, and manifolds with fibrations over hyperbolic base orbifolds have unique Seifert-fibered structures [32, Theorem 3.8].

Observation 4.5. The set of manifolds $\left\{M_{i, 0}\right\}$ form a $\mathbb{K}_{4}$ in $\mathcal{B}$.

We will now construct a $\mathbb{K}_{\infty}$ which consists of infinitely many of these $\mathbb{K}_{4}$. Note that if we add 1 to each Seifert invariant of each exceptional fiber above, we get another $\mathbb{K}_{4}$. Each new manifold is distinct from the manifolds in the previous $\mathbb{K}_{4}$ since the sum of its Seifert invariants is not equal to any vertex in the original. Each vertex in the new $\mathbb{K}_{4}$ is connected to each vertex of the previous $\mathbb{K}_{4}$ as, for example, $S F S\left(S^{2} ; a_{1} / b_{1}+1, a_{2} / b_{2}+1, a_{3} / b_{3}+1\right) \equiv S F S\left(S^{2} ; a_{1} / b_{1}+3, a_{2} / b_{2}, a_{3} / b_{3}\right) \equiv$ $S F S\left(S^{2} ; a_{1} / b_{1}, a_{2} / b_{2}+3, a_{3} / b_{3}\right) \equiv S F S\left(S^{2} ; a_{1} / b_{1}, a_{2} / b_{2}, a_{3} / b_{3}+3\right)$. Dehn surgery along one of the exceptional fibers can result in any manifold which is a vertex of the original $\mathbb{K}_{4}$. Continuing this way, we have a $\mathbb{K}_{\infty}$, parametrized by $(i, j)$, where $i \in\{1,2,3,4\}$ and $j \in \mathbb{N}$. Specifically, $M_{i, j}$ is as follows:

$$
\begin{gathered}
\left\{M_{1, j}=S F S\left(S^{2} ; a_{2} / b_{2}+j, a_{3} / b_{3}+j, a_{4} / b_{4}+j\right),\right. \\
M_{2, j}=S F S\left(S^{2} ; a_{1} / b_{1}+j, a_{3} / b_{3}+j, a_{4} / b_{4}+j\right), \\
M_{3, j}=S F S\left(S^{2} ; a_{1} / b_{1}+j, a_{2} / b_{2}+j, a_{4} / b_{4}+j\right), \\
\left.M_{4, j}=S F S\left(S^{2} ; a_{1} / b_{1}+j, a_{2} / b_{2}+j, a_{3} / b_{3}+j\right)\right\} .
\end{gathered}
$$

Assume this $\mathbb{K}_{\infty}$ comes from filling a one cusped manifold $M$. First, $M$ must be irreducible. Indeed, if it were reducible, there would be a two-sphere that did not bound a ball in $M$. If the sphere is non-separating it will remain non-separating in any filling. If it is separating, there is at most one filling of a knot in a ball which will make it a ball [11.

Next we observe that each $M_{i, j}$ is a small Seifert-fibered space and in particular non-Haken. We claim that we may assume $M$ does not contain an essential torus. Indeed, if $M$ does contain an essential torus $T$, then that torus compresses in infinitely many fillings. Infinitely many fillings cannot be pairwise distance 1 and thus by [7, Theorem 2.01], $T$ and $\partial M$ cobound a cable space, $C$. Surgeries on cable spaces are well understood. As in [4, p 179], the filling of the cable space is either reducible (only along the cabling slope), a solid torus, or a manifold with an incompressible boundary torus. Since $T$ is compressible in the fillings, $T$ bounds a 
solid torus in each of the filled manifolds. Therefore, we can replace filling along $\partial M$ by filling along the torus boundary of $M \backslash C$ and get the same set of manifolds.

Thus we may assume $M$ does not contain an essential torus and since it is irreducible $M$ is geometric.

If $M$ is not hyperbolic, it is a Seifert-fibered space containing no essential tori. Thus $M$ must be Seifert-fibered over the disk with at most two exceptional fibers. Each $M_{i, j}$ admits a unique Seifert fibration (see [32, Theorem 3.8]). Any choice of two elements $\left\{a_{i} / b_{i}\right\}$ to label the exceptional fibers of $M$ will disagree with two exceptional fibers in one of the $M_{i}$, which is a contradiction to the existence of such an $M$. Thus $M$ must be hyperbolic. However, there are infinitely many Seifert-fibered manifolds that come from surgery on $M$. This contradicts Thurston's Hyperbolic Dehn Surgery Theorem [33, Theorem 5.8.2].

\section{Seifert-Fibered SPACES AND Solv manifolds}

Before we discuss the hyperbolic part of the graph, we briefly discuss other geometries and Seifert-fibered spaces. By compiling work of Montesinos and Dunbar, we can obtain upper bounds for any non-hyperbolic geometric manifold. The general idea is that "simpler" manifolds lie close to $S^{3}$. We begin with a theorem of Montesinos [23. $\chi(F)$ is the Euler characteristic of $F$.

Theorem 5.1 (Montesinos). Let $M$ be a closed, orientable Seifert-fibered space over the surface $F$ with $n$ exceptional fibers.

(1) If $F$ is orientable, then $p_{L}\left(M, S^{3}\right) \leq 3-\chi(F)+n$.

(2) If $F$ is non-orientable, then $p_{L}\left(M, S^{3}\right) \leq 5-2 \chi(F)+n$.

This theorem follows from the discussion in [23, Chapter 4] (see specifically Figure 12 in that chapter). Since each link in that figure has a component labeled by $\frac{1}{b}$, mild Kirby Calculus can be applied to the links in that figure to obtain a link with one fewer component.

To understand the geometric non-hyperbolic manifolds, it remains to investigate Solv manifolds, which are either torus bundles over $S^{1}$ or the union of twisted $I$ bundles over the Klein bottle (see [32, Theorem 4.17]). Work of Dunbar provides an orbifold analog to this statement, namely if $Q$ is an orientable Solv orbifold, $Q$ is either a manifold as above or an orbifold with fiber $S^{2}(2,2,2,2)$ over $S^{1}$ or the union of twisted $I$ bundles with fiber $S^{2}(2,2,2,2)$ (see [9, Propostion 1.1]). Using these two results, we can obtain the following:

Proposition 5.2. (1) If $M$ is a Solv torus bundle, then $p_{L}\left(M, S^{3}\right) \leq 5$.

(2) If $M$ is the union of twisted I bundles over the Klein bottle admitting an orientable Solv structure, then $p_{L}\left(M, S^{3}\right) \leq 3$.

Proof. (1) By 9], $M$ admits a 2-fold quotient $Q$ such that the base space of $Q$ is $S^{1} \times S^{2}$ and the singular locus is a four strand braid $B$. Although that paper is careful to classify such braids, the details will not be relevant to this argument. Using the Montesinos trick, we have a sequence of tangle replacements to get from $Q$ to the trivial two strand braid in $S^{1} \times S^{2}$. The first two replacements of this sequence are shown in Figure 3(a) The resulting link is two-bridge and therefore a single rational tangle replacement yields the unknot. The trivial two strand braid can be obtained from a single rational tangle replacement on the unknot. Hence, $p_{L}\left(M, S^{1} \times S^{2}\right) \leq 4$ and $p_{L}\left(M, S^{3}\right) \leq 5$. 


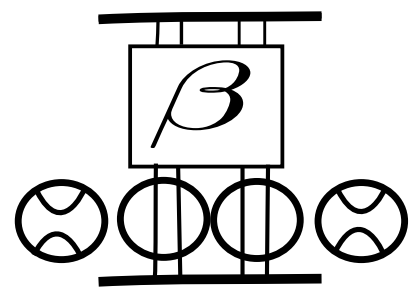

(a) Replacement of these rational tangles gives a nullhomotopic two-bridge link in $S^{2} \times S^{1}$.

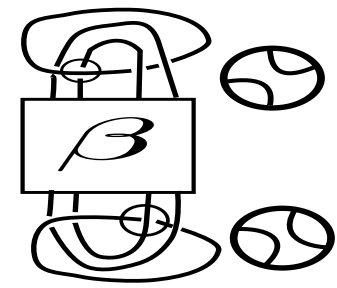

(b) Replacement of these rational tangles gives a null-homotopic two-bridge link in $S^{3}$

FiguRE 3. Rational tangle replacements on quotients of Solv manifolds.

(2) Let $M$ be the union of twisted $I$ bundles over the Klein bottle admitting an orientable Solv structure. Then $M$ is the 2 -fold quotient of $\tilde{M}$ a Solv torus bundle. Moreover, $\pi_{1}(\tilde{M})$ is the index 2 subgroup of $\pi_{1}(M)$ elements that preserve the orientation of every fiber of $M$ and we may consider $\pi_{1}(M)=\left\{\pi_{1}(\tilde{M}), \rho \pi_{1}(\tilde{M})\right\}$ where $\rho$ is the composition of a translation $t$ in a fiber and a symmetry of Solv taking the form $\langle x, y, z\rangle \rightarrow\langle y, x,-z\rangle$ or $\langle-y,-x,-z\rangle$.

Denote by $Q \cong M /\langle t\rangle$ the 2-fold quotient of $M$ by $t$. The base space of $Q$ is $S^{3}$ and the singular set is isotopic to the link picture in Figure 3(b), The rational tangle replacements in that figure yield a two-bridge link and so the double branched cover of the resulting link is a lens space. A lens space is path length one from $S^{3}$, completing the proof.

Immediately from this section, we have that if $M$ is a closed orientable 3-manifold which admits a Nil, $\mathbb{E}^{3}, S^{2} \times \mathbb{R}, S^{3}$ or Solv geometry, then $p_{L}\left(M, S^{3}\right) \leq 5$. However, these upper bounds are not in general known to be sharp. The reader is referred to Margaret Doig's work [8] for a more comprehensive treatment of which manifolds admitting an elliptic geometric structure can be obtained from surgery along a knot in $S^{3}$.

\section{THE SUBGRAPH FOR HYPERBOLIC MANIFOLDS}

Definition 6.1. Let $\mathcal{B}_{H}$ be the subgraph of $\mathcal{B}$ such that the vertices correspond to closed hyperbolic 3-manifolds, and there is an edge between two vertices $v_{M}$ and $v_{N}$ exactly when there is a one-cusped hyperbolic 3-manifold $P$ with two fillings homeomorphic to $M$ and $N$.

Note that there is not necessarily a hyperbolic Dehn surgery between $M$ and $N$ in our definition. For example, the surgery knot might not be represented by embedded geodesics in $M$ and $N$.

As mentioned above in $\$ 4$ this part of the graph has the nice property that if two different $K_{\infty}$ graphs that arise as $M(K)$ and $M\left(K^{\prime}\right)$ intersect, they must do so in finitely many vertices. We conjecture that the combinatorics of this subgraph may reveal more of geometry and topology than in the full graph. For the same reasons as $\mathcal{B}, \mathcal{B}_{H}$ is infinite valence and infinite diameter. We show here that it is connected, using the work of Myers. Let $Y$ be a compact orientable 3-manifold, possibly with boundary. Following Myers, we say that $Y$ is excellent if it is irreducible 
and boundary irreducible, not a 3-ball, every properly embedded incompressible surface of zero Euler characteristic is isotopic into the boundary, and it contains a two-sided properly embedded incompressible surface. These manifolds are known by Thurston [34, Theorem 1.2] to admit hyperbolic structures. By slight abuse of notation, if a properly embedded 1-manifold $K \subset Y$ has an excellent exterior, we will call $K$ excellent.

Theorem 6.2 (Myers). Let $M$ be a compact connected 3-manifold whose boundary does not contain 2-spheres or projective planes. Let $J$ be a compact properly embedded 1-manifold in $M$. Then $J$ is homotopic rel $\partial J$ to an excellent 1-manifold $K$.

For the following lemma, we observe Myers' notation in stating the following technical lemma. Namely, let $X$ be a 3 -manifold and $F$ be a compact, (possibly disconnected) properly embedded two-sided surface in $X$. Let $Y$ be the manifold obtained by cutting along $F$ and let $F_{1}, F_{2} \subset \partial Y$ be such that identifying $F_{1}$ and $F_{2}$ yields $X$.

Lemma 6.3 (Myers' Gluing Lemma, [26, Lemma 2.1]). If each component of $Y$ is excellent, $F_{1} \cup F_{2}$ and $c l\left(\partial Y \backslash\left(F_{1} \cup F_{2}\right)\right)$ are incompressible in $Y$, and each component of $F_{1} \cup F_{2}$ has negative Euler characteristic, then $X$ is excellent.

Theorem 6.4. Suppose $M_{0}$ and $M_{n}$ are closed hyperbolic 3-manifolds such that the associated vertices $v_{M_{0}}$ and $v_{M_{n}}$ are connected via a path of length $n$ in $\mathcal{B}$. Then $v_{M_{0}}$ and $v_{M_{n}}$ are connected via a path of length $n+2$ in $\mathcal{B}_{H}$.

Proof. Observe that, under this hypothesis, there is an $n$-component link, $\left\{a_{1}, \ldots\right.$, $\left.a_{n}\right\}$ in $M_{0}$ and closed manifolds $M_{1}, \ldots, M_{n}$ such that

$$
M_{i}=M_{0}\left(\left\{a_{1}, \ldots, a_{n}\right\} ;\left(\beta_{1}, \beta_{2}, \ldots, \beta_{i}, \alpha_{i+1}, \ldots, \alpha_{n}\right)\right), i \in\{0, . ., n\} .
$$

We will find a knot $k$ in $M_{0} \backslash\left\{a_{1}, \ldots, a_{n}\right\}$ and a slope $r$ such that the closed manifolds

$$
N_{i}=M_{0}\left(\left\{a_{1}, \ldots, a_{n}, k\right\} ;\left(\beta_{1}, \ldots, \beta_{i}, \alpha_{i+1}, \ldots, \alpha_{n}, r\right)\right), i \in\{0, \ldots, n\},
$$

are hyperbolic. Each $N_{i}$ is obtained from $M_{i}$ via Dehn surgery on $k$ with slope $r$. The knot $k$ and slope $r$ will also have the property that the one-cusped manifolds

$$
\begin{gathered}
P_{i}=M_{0}\left(\left\{a_{1}, \ldots, a_{n}, k\right\} ;\left(\beta_{1}, \ldots, \beta_{i-1},-, \alpha_{i+1}, \ldots, \alpha_{n}, r\right)\right), \\
Q_{0}=M_{0}\left(\left\{a_{1}, \ldots, a_{n}, k\right\} ;\left(\alpha_{1}, \ldots, \alpha_{n},-\right)\right),
\end{gathered}
$$

and

$$
Q_{n}=M_{0}\left(\left\{a_{1}, \ldots, a_{n}, k\right\} ;\left(\beta_{1}, \ldots, \beta_{n},-\right)\right),
$$

are hyperbolic. We will use Myers' Theorem 6.2 and Lemma 6.3, stated above. We will also use the fact, proven in Lemma 6.5, that, given a $T^{2} \times I$ and two slopes $x$ and $y$ on $T^{2} \times\{0\}$, there is an arc $A$ in $T^{2} \times I$ with endpoints on $T^{2} \times\{1\}$ such that the exterior $H_{A}$ of $A$ in $T^{2} \times I$ is excellent. Furthermore, the results of Dehn filling $H_{A}$ along the slopes $x$ and $y$ are excellent.

Now we prove the existence of a knot $k$ in the exterior of the link $\left\{a_{1}, \ldots, a_{n}\right\}$ in $M_{0}$ with the desired properties. First fix a homeomorphism $h_{i}$ of a neighborhood $N\left(\partial N\left(a_{i}\right)\right)$ of each $\partial N\left(a_{i}\right)$ with $T^{2} \times I$. For each component $a_{i}$, we construct an arc $A_{i}$ in $T^{2} \times I$ such that: (i) $\partial A_{i} \subset T^{2} \times\{1\}$; (ii) the exterior of $A_{i}$ in $T^{2} \times I$ 
is excellent; and (iii) the results of filling the exterior of $A_{i}$ along the slopes $h_{i}\left(\alpha_{i}\right)$ and $h_{i}\left(\beta_{i}\right)$ on $T^{2} \times\{0\}$ are excellent. This is done in Lemma 6.5 below. Now by Myers' Theorem, stated as Theorem 6.2 above, there is an excellent collection of $\operatorname{arcs}\left\{B_{i}\right\}$ in $M_{0} \backslash\left\{N\left(N\left(a_{i}\right)\right)\right\}$ such that $B_{i}$ connects an endpoint of $A_{i}$ to one of $A_{i+1} \bmod n$. Then we claim the following:

(1) $k=\bigcup_{n}\left(A_{i} \cup B_{i}\right)$ is an excellent knot in $M_{0} \backslash\left\{N\left(a_{i}\right)\right\}$.

(2) The result of filling along any choice of $\alpha_{i}$ or $\beta_{i}$ for any subset of the $a_{i}$ is excellent.

The fact that the union of arcs in (1) above is a knot follows from the recipe. The fact that the exterior in (1) is excellent follows from Myers' Lemma 6.3 above and the fact that each $T^{2} \times I \backslash N\left(A_{i}\right)$ is excellent and that the exterior of the union of the $B_{i}$ is excellent. Similarly, since each $T^{2} \times I \backslash N\left(A_{i}\right)$ filled along $\alpha_{i}$ or $\beta_{i}$ is excellent, Myers' Gluing Lemma 6.3 yields that filling any subset of the $a_{i}$ along $\alpha_{i}$ or $\beta_{i}$ is excellent. Thus, in particular, $Q_{0}$ and $Q_{n}$ above are hyperbolic.

Let $k$ be a knot in $M_{0} \backslash\left\{N\left(a_{i}\right)\right\}$ having property (1) above. Choose a slope $r$ on $\partial N(k)$ such that $r$ lies outside of the finite set of slopes that makes any one of the closed manifolds $N_{i}$ or the cusped manifolds $P_{i}$ not hyperbolic.

Then the path $M_{0}, Q_{0}, N_{0}, P_{1}, N_{1}, P_{2}, \ldots, N_{n}, Q_{n}, M_{n}$ is a path in $\mathcal{B}_{H}$ connecting $v_{M_{0}}$ and $v_{M_{n}}$. Here the $M_{i}$ and $N_{i}$ are closed hyperbolic manifolds (represented by vertices in $\mathcal{B}_{H}$ ) and the $P_{i}$ and $Q_{i}$ are cusped hyperbolic manifolds (represented by edges in $\mathcal{B}_{H}$ ).

Lemma 6.5. Given $T^{2} \times[0,1]$ and two isotopy classes of curves $x$ and $y$ on $T^{2} \times\{0\}$, there is an arc $A$ with endpoints on $T^{2} \times\{1\}$ such that:

(1) $T^{2} \times I \backslash N(A)$ is excellent.

(2) The results of filling $T^{2} \times I \backslash N(A)$ along the slopes $x$ and $y$ are excellent.

Proof. By Myers' Theorem 6.2, there exists an arc $E$ in $T^{2} \times I$ with endpoints on $T^{2} \times\{1\}$ such that the exterior $T^{2} \times I \backslash N(E)$ is excellent. The arc we will use is $E$, wrapped around enough to make filling along two specified slopes $x$ and $y$ hyperbolic. We detail this wrapping around below.

Fix $T^{2} \times I$ up to isotopy. Let $m$ be an oriented slope on $T^{2} \times\{0\}$. Let $A_{m}$ be an essential annulus bounded by $m$ and a curve $m^{\prime}$ on $T^{2} \times\{1\}$. Let $l$ be a slope that has intersection number 1 with $m$. There are homeomorphisms $f_{m}, f_{l}: T^{2} \times I \rightarrow T^{2} \times I$ obtained by cutting along $A_{m}$ and $A_{l}$, twisting once, and then gluing back by the identity on this annulus. We twist so that an oriented $f_{m}(p m+q l)=p m+(q+1) l$ and $f_{l}(p m+q l)=(p+1) m+q l$, in the original isotopy class of $T^{2} \times I$. Furthermore, given an $n \in \mathbb{N}$ and an oriented slope $t$, there is an $f$, which is a composition of $f_{m}$ and $f_{l}$ such that the oriented intersection of $t$ and $m$ and $t$ and $l$ is larger than $n$.

Now let $H_{E}$ be the exterior of $E$ in $T^{2} \times I$. There is a subsurface $D=T^{2} \times$ $\{1\} \backslash N(\partial E)$ of the boundary such that it and its complement are incompressible in $H_{E}$. Thus we may apply Myers' Gluing Lemma (Lemma 6.3) to the double along $D, D H_{E}$ and conclude that it is excellent, hence hyperbolic. The manifold $D H_{E}$ is the exterior of a knot in $T^{2} \times[0,2]$. We say that filling along the components $T^{2} \times\{0\}$ and $T^{2} \times\{2\}$ such that the filling is the double along $D$ of a filling of $H_{E}$ is a symmetric filling. Then, by Thurston's Hyperbolic Dehn Surgery Theorem [33. Theorem 5.8.2], all but finitely many symmetric fillings of $D H_{E}$ are hyperbolic. (Note that the filling curves have the same length in the complete structure on $D H_{E}$.) The maps $f_{m}$ and $f_{l}$ extend naturally to $D H_{E}$ (by restriction to $H_{E}$ and 
doubling) and take symmetric slopes to symmetric slopes. Thus there is a map $f: D H_{E} \rightarrow D H_{E}$, which can be taken to be of the form $f_{m}^{n} f_{l}^{p}$, such that filling $D H_{E}$ symmetrically along $f^{-1}(x)$ and $f^{-1}(y)$ is hyperbolic. Then the arc $A=f(E)$ in $T^{2} \times I$ has the property that filling along $x$ and $y$ is hyperbolic. Indeed, doubling the exterior of $A$ results in $f\left(D H_{E}\right)$ which is hyperbolic when symmetrically Dehn filled along $x$ and $y$.

\section{Obstructions to $\delta$-HyPerbolicity}

We recall the following definitions. A geodesic metric space is $\delta$-hyperbolic if every geodesic triangle is " $\delta$ thin", that is, every side is contained in a $\delta$-neighborhood of the union of the other two sides. Two metric spaces $X, Y$ with metrics $\mu_{X}, \mu_{Y}$ are quasi-isometric if there exists a function $f: X \rightarrow Y$ and $A \geq 1, B \geq 0, C \geq 0$ such that (1) for all $x_{1}, x_{2} \in X, \frac{1}{A} \mu_{Y}\left(f\left(x_{1}, x_{2}\right)\right)-B \leq \mu_{X}\left(x_{1}, x_{2}\right) \leq A \cdot \mu_{Y}\left(f_{1}\left(x_{1}, x_{2}\right)\right)+B$ and (2) every point of $Y$ lies in the $C$-neighborhood of $f(X)$. A $k$-quasi-flat in a metric space $X$ is a subset of $X$ that is quasi-isometric to $\mathbb{E}^{k}$. In this section we will construct quasi-flats in $\mathcal{B}$ and $\mathcal{B}_{H}$, showing that these spaces are not $\delta$-hyperbolic.

We will need to compute the exact distance in some simple examples. To do so, we first give a method for a lower bound on the distance.

Lemma 7.1. Let $M_{1}$ and $M_{2}$ be closed orientable 3 -manifolds and let $0 \leq m \leq n$ and $p$ a prime. If $\pi_{1}\left(M_{1}\right) \rightarrow(\mathbb{Z} / p \mathbb{Z})^{n}$ and $\pi_{1}\left(M_{2}\right) \rightarrow(\mathbb{Z} / p \mathbb{Z})^{m}$ but $\pi_{1}\left(M_{2}\right) \not \nrightarrow$ $(\mathbb{Z} / p \mathbb{Z})^{m+1}$, then

$$
p_{L}\left(M_{1}, M_{2}\right) \geq n-m .
$$

Proof. Let $K$ be a knot in a closed manifold $M$, and let $w$ be a word in $\pi_{1}(M(K ;-))$. We claim that if $\phi: \pi_{1}(M(K ;-)) \rightarrow(\mathbb{Z} / p \mathbb{Z})^{n}$ is a surjection, then $\phi$ induces a surjection $\phi^{\prime}$ from $\pi_{1}(M(K ;-)) /\langle\langle w\rangle\rangle$ to $(\mathbb{Z} / p \mathbb{Z})^{n}$ or $(\mathbb{Z} / p \mathbb{Z})^{n-1}$. Indeed, the image of $w$ under $\phi$ is either trivial or non-trivial. If it is trivial, then $\phi$ induces a surjection $\phi^{\prime}: \pi_{1}(M(K ;-)) /\langle\langle w\rangle\rangle$ to $(\mathbb{Z} / p \mathbb{Z})^{n}=(\mathbb{Z} / p \mathbb{Z})^{n} /\langle\langle\phi(w)\rangle\rangle$. If $\phi(w)$ is non-trivial, then it is order $p$ in $(\mathbb{Z} / p \mathbb{Z})^{n}$, since every element is order $p$. Then there is a minimal generating set of $(\mathbb{Z} / p \mathbb{Z})^{n}$ where $\phi(w)$ is a basis element. Then $\phi^{\prime}: \pi_{1}(M(K ;-)) /\langle\langle w\rangle\rangle \rightarrow(\mathbb{Z} / p \mathbb{Z})^{(n-1)}=(\mathbb{Z} / p \mathbb{Z})^{n} /\langle\langle\phi(w)\rangle\rangle$ is a surjection. This proves the claim.

We note that if $\pi_{1}(M(K ;-)) /\langle\langle w\rangle\rangle$ surjects $(\mathbb{Z} / p \mathbb{Z})^{n}$, then $\pi_{1}(M(K ;-))$ does as well, since there is a presentation of the two groups which differs only by the addition of a relation. Then the claim implies that the maximum $n$ such that $\pi_{1}(N)$ surjects $(\mathbb{Z} / p \mathbb{Z})^{n}$ can change by at most 1 under the operation of Dehn surgery along a knot in $N$, and the lemma follows.

Theorem 7.2. $\mathcal{B}$ contains a 2-quasi-flat. Hence $\mathcal{B}$ is not $\delta$-hyperbolic.

Proof. For each $n$, let $U_{n}$ be the unlink in $S^{3}$ with $n$ components with the natural homological framing. Then we will consider the manifolds:

$$
\begin{gathered}
A_{j}=S^{3}\left(U_{2 n} ;\left(\frac{p_{1}}{1}, \frac{p_{1}}{1}, \ldots \frac{1}{0}, \frac{1}{0}\right)\right), \\
B_{k}=S^{3}\left(U_{2 n} ;\left(\frac{1}{0}, \frac{1}{0}, \ldots, \frac{p_{1} p_{2}}{1}, \frac{p_{1} p_{2}}{1}\right)\right), \text { and } \\
C_{j, k}=S^{3}\left(U_{2 n} ;\left(\frac{p_{1}}{1}, \ldots \frac{1}{0}, \frac{1}{0}, \frac{p_{1} p_{2}}{1}, \frac{p_{1} p_{2}}{1}, \ldots \frac{1}{0}, \frac{1}{0}\right)\right) .
\end{gathered}
$$

In other words, the surgeries on the first $n$ components are either $\frac{p_{1}}{1}$ or $\frac{1}{0}$, with surgery on the first $j$ components being $\frac{p_{1}}{1}$. Let $p_{1}$ and $p_{2}$ be distinct primes. The 


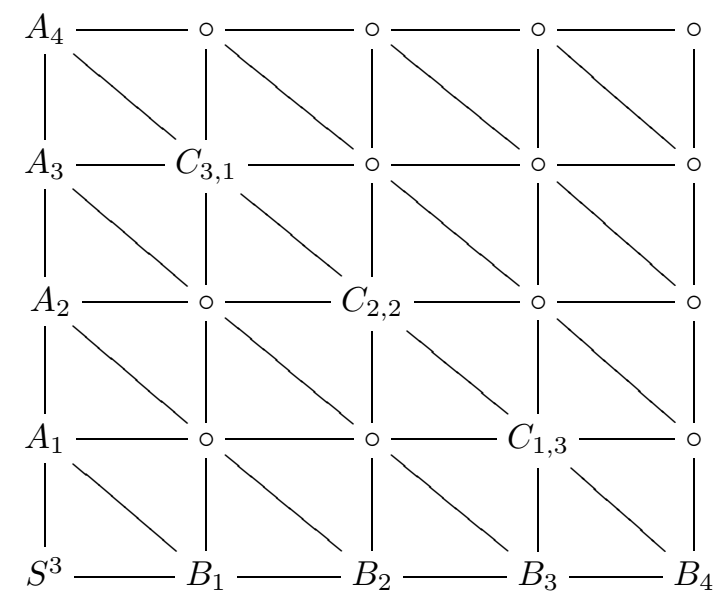

Figure 4. This represents a 2 -quasi-flat in $\mathcal{B}$. In particular, the distance between the manifolds in the figure can be determined using the edges in the figure.

surgeries on the second $n$ components are either $\frac{p_{1} p_{2}}{1}$ or $\frac{1}{0}$, with the first $k$ being $\frac{p_{1} p_{2}}{1}$. The number of non-trivial fillings of $A_{j}$ is $j$, of $B_{k}$ is $k$, and of $C_{j, k}$ is $j+k$. Thus we can take $n$ as large as needed and these are still well defined.

Then:

$$
\begin{gathered}
H_{1}\left(A_{j}, \mathbb{Z}\right)=\left(\mathbb{Z} / p_{1} \mathbb{Z}\right)^{j}, \\
H_{1}\left(B_{k}, \mathbb{Z}\right)=\left(\mathbb{Z} / p_{1} p_{2} \mathbb{Z}\right)^{k}, \text { and } \\
H_{1}\left(C_{j, k}, \mathbb{Z}\right)=\left(\mathbb{Z} / p_{1} \mathbb{Z}\right)^{j} \oplus\left(\mathbb{Z} / p_{1} p_{2} \mathbb{Z}\right)^{k} .
\end{gathered}
$$

Then, by repeated use of Lemma 7.1, since every map to an abelian group factors through the homology, the distances between these manifolds are as in the diagram.

Using the same methods, we can show the following.

Theorem 7.3. $\mathcal{B}$ has a 4-quasi-flat based at $S^{3}$. Furthermore, $\mathcal{B}$ has a 4-quasi-flat based at each vertex $v_{M}$.

Proof. By choosing four distinct primes $p_{1}, p_{2}, p_{3}$ and $p_{4}$, the graph $\mathcal{B}$ can be seen to exhibit a large quasi-flat based at $S^{3}$. The vertices of such a quasi-flat are:

$$
\begin{gathered}
E_{j}=S^{3}\left(U_{2 n} ;\left(\frac{p_{1}}{1}, \frac{p_{1}}{1}, \ldots, \frac{1}{0}, \frac{1}{0}\right)\right), \\
N_{j}=S^{3}\left(U_{2 n} ;\left(\frac{1}{0}, \frac{1}{0}, \ldots, \frac{p_{1} p_{2}}{1}, \frac{p_{1} p_{2}}{1}\right)\right), \\
W_{j}=S^{3}\left(U_{2 n} ;\left(\frac{p_{1} p_{2} p_{3}}{1}, \frac{p_{1} p_{2} p_{3}}{1}, \ldots, \frac{1}{0}, \frac{1}{0}\right)\right), \text { and } \\
S_{j}=S^{3}\left(U_{2 n} ;\left(\frac{1}{0}, \frac{1}{0}, \ldots, \frac{p_{1} p_{2} p_{3} p_{4}}{1}, \frac{p_{1} p_{2} p_{3} p_{4}}{1}\right)\right) .
\end{gathered}
$$

In fact, if the manifolds $E_{j}, N_{j}, W_{j}$ and $S_{j}$ are connect summed with a given closed orientable manifold $M$, then by the same homology argument as above, there is a quasi-flat based at $M$.

Remark 7.4. This construction can be adapted to construct $k$-quasi-flats for arbitrarily large $k$. 
The behavior of homology under Dehn filling is a key property of the $2 n$ component split link in the argument above. The pairwise linking number of the components of that link is 0 . The rest of this section will be devoted to finding a $2 n+1$ component link that has similar behavior. First, we construct a hyperbolic link where each one of the pairwise linking numbers is 0 . This is accomplished as follows:

Lemma 7.5. There is a knot $K$ in the complement of the $m$ component split link $U_{m}$ such that $S^{3}\left(U_{m} \cup K ;-\right)$ is hyperbolic and all components have pairwise linking number 0 .

Proof. The proof is similar to the methods in 96 . Consider the link exterior $M=$ $S^{3}\left(U_{m} ;-\right)$ and label each boundary component by $C_{i}$. Let $H_{i}$ be a neighborhood of each $C_{i}$ in $M$. By [26], we can drill out a set of excellent $\operatorname{arcs} a_{i} 1 \leq i<m$ from $N=M \backslash \bigcup H_{i}$ such that $a_{i}$ connects a neighborhood of the $i$ th component with the $(i+1)$ st component and $a_{m}$ connects the last component to the first. Furthermore, orient each $a_{i}$ such that $a_{i}$ is based on a neighborhood of the $i$ th component. For convenience denote the last arc by $a_{0}$ and $a_{m}$. If $D_{i}$ is the disk in $M$ with $C_{i}$ as a boundary, the union of the the arcs in $N$ will have oriented intersection number $\lambda_{i}$ with $D_{i} \cap N$.

Let $A_{i}=H_{i} \cap D_{i}$. With a slight abuse of notation we consider $H_{i}$ as homeomorphic to $T^{2} \times I$ with the marked annulus $A_{i}$ embedded in it. Again by [26], we can drill out an excellent arc from $H_{i}$ in any homotopy class, and hence with any intersection number with $A_{i}$, connecting the endpoints of $a_{i}$ and $a_{i-1}$. Here, we choose $-\lambda_{i}$ to be this intersection number.

Let $K=\bigcup a_{i}$ be an oriented knot in $S^{3}\left(L_{m} ;-\right)$. For each component $C_{i}$ of $L_{m}$, the disk $D_{i}$ is also a Seifert surface for $C_{i}$. Thus, the pairwise linking number of $C_{i}$ and $K$ is the oriented intersection number of $K$ with $D_{i}, 0=\lambda_{i}-\lambda_{i}$.

In the above proof, there is a special component of the link, $K$, such that drilling out $K$ from $S^{3}\left(L_{m} ;-\right)$ is hyperbolic. We call this component of the link $L_{m} \cup K$ the Myers component. For a general $n$-component link an $(n-1)$-component must be specified to determine the Myers component.

Theorem 7.6. $\mathcal{B}_{H}$ is not $\delta$-hyperbolic.

Proof. As above, we construct a quasi-flat. Using the link $L^{\prime}=L_{n} \cup K$ as in Lemma 7.5. we have that $S^{3}\left(L^{\prime} ;-\right)$ is hyperbolic and each pair of components has linking number 0 . This condition implies that $K$, an embedded curve, is null homologous in

$$
S^{3}\left(L^{\prime} ;\left(\frac{r_{1}}{s_{1}}, \ldots, \frac{r_{n}}{s_{n}}, \frac{1}{0}\right)\right) \cong L\left(r_{1}, s_{1}\right) \# \ldots \# L\left(r_{n}, s_{n}\right),
$$

since the homology class of $K$ is determined by the sum of the oriented $\bmod r_{i}$ intersection number with the Seifert surface of the $i$ th component of $L_{n}$. One can observe this directly by consideration of $K$ as a curve in $S^{3}\left(L^{\prime} ;\left(\frac{1}{0}, \ldots, \frac{1}{0}, \frac{r_{i}}{s_{i}}, \frac{1}{0}, \ldots, \frac{1}{0}, \frac{1}{0}\right)\right)$ $\cong L\left(r_{i}, s_{i}\right)$.

Thus,

$$
H_{1}\left(S^{3}\left(L^{\prime} ;\left(\frac{r_{1}}{s_{1}}, \ldots, \frac{r_{n}}{s_{n}}, \frac{1}{q^{\prime}}\right)\right), \mathbb{Z}\right)=\mathbb{Z} / r_{1} \mathbb{Z} \oplus \cdots \oplus \mathbb{Z} / r_{n} \mathbb{Z}
$$

and so we can choose surgery coefficients such that the homology of the fillings behaves analogously to the manifolds $A_{j}, B_{k}$, and $C_{j, k}$ as in the proof of Theorem 7.2 . 
Finally, we remark that choosing sufficiently large choices of primes $p_{1}$ and $p_{2}$ and a large choice of $q^{\prime}$, the manifolds obtained by filling the first $n$ components of $S^{3}\left(L^{\prime} ;\left(-, \ldots,-, \frac{1}{q^{\prime}}\right)\right)$ by either $\frac{p_{1}}{1}$ or $\frac{p_{1} p_{2}}{1}$ is hyperbolic by Thurston's Hyperbolic Dehn Surgery Theorem [33, Theorem 5.8.2].

\section{ACKNOWLEDGEMENTS}

Both authors have benefited from many conversations with colleagues. We would particularly like to thank Margaret Doig for suggesting that we look at the manifolds in [13. We are also grateful to Steven Boyer, Nathan Dunfield, Marc Lackenby, Tao Li, Luisa Paoluzzi, and Richard Webb. The first author was partially supported by ARC Discovery Grant DP130103694 and the Max Planck Institute for Mathematics. The second author was partially supported through NSF Grant 1207644 and a Tufts University Faculty Research Award. We particularly thank the referee for many helpful comments.

\section{RefERENCES}

[1] Matthias Aschenbrenner and Stefan Friedl, 3-manifold groups are virtually residually $p$, Mem. Amer. Math. Soc. 225 (2013), no. 1058, viii+100, DOI 10.1090/S0065-9266-2013-00682-X. MR3100378

[2] David Auckly, An irreducible homology sphere which is not Dehn surgery on any knot, preprint available at http://www.math.ksu.edu/ dav/.

[3] David Auckly, Surgery numbers of 3-manifolds: a hyperbolic example, Geometric topology (Athens, GA, 1993), AMS/IP Stud. Adv. Math., vol. 2, Amer. Math. Soc., Providence, RI, 1997, pp. 21-34. MR1470719 (98d:57030)

[4] Steven Boyer, Dehn surgery on knots, Handbook of geometric topology, North-Holland, Amsterdam, 2002, pp. 165-218. MR 1886670 (2003f:57030)

[5] Steven Boyer and Daniel Lines, Surgery formulae for Casson's invariant and extensions to homology lens spaces, J. Reine Angew. Math. 405 (1990), 181-220. MR.1041002 (91e:57026)

[6] Marc Culler and Nathan M. Dunfield, SnapPy, a computer program for studying the geometry and topology of 3-manifolds. available at http://snappy.computop.org.

[7] Marc Culler, C. McA. Gordon, J. Luecke, and Peter B. Shalen, Dehn surgery on knots, Ann. of Math. (2) 125 (1987), no. 2, 237-300, DOI 10.2307/1971311. MR881270 (88a:57026)

[8] Margaret I. Doig, Finite knot surgeries and Heegaard Floer homology, Algebr. Geom. Topol. 15 (2015), no. 2, 667-690, DOI 10.2140/agt.2015.15.667. MR 3342672

[9] William D. Dunbar, Classification of solvorbifolds in dimension three. I, Braids (Santa Cruz, CA, 1986), Contemp. Math., vol. 78, Amer. Math. Soc., Providence, RI, 1988, pp. 207-216, DOI 10.1090/conm/078/975080. MR975080(89k:57027)

[10] C. McA. Gordon, Dehn surgery and satellite knots, Trans. Amer. Math. Soc. 275 (1983), no. 2, 687-708, DOI 10.2307/1999046. MR682725 (84d:57003)

[11] C. McA. Gordon and J. Luecke, Knots are determined by their complements, Bull. Amer. Math. Soc. (N.S.) 20 (1989), no. 1, 83-87, DOI 10.1090/S0273-0979-1989-15706-6. MR.972070 (90a:57006b)

[12] C. McA. Gordon and J. Luecke, Toroidal and boundary-reducing Dehn fillings, Topology Appl. 93 (1999), no. 1, 77-90, DOI 10.1016/S0166-8641(97)00258-7. MR 1684214 (2000b:57030)

[13] Joshua Evan Greene and Liam Watson, Turaev torsion, definite 4-manifolds, and quasi-alternating knots, Bull. Lond. Math. Soc. 45 (2013), no. 5, 962-972, DOI 10.1112/blms/bds096. MR3104988

[14] Michael Gromov, Hyperbolic manifolds (according to Thurston and Jørgensen), Bourbaki Seminar, Vol. 1979/80, Lecture Notes in Math., vol. 842, Springer, Berlin-New York, 1981, pp. 40-53. MR636516 (84b:53046)

[15] Alan E. Hatcher, Notes on basic 3-manifold topology, available at http://www.math.cornell. edu/ hatcher/3M/3Mdownloads.html, 2000.

[16] Damian Heard, Orb, available at www.ms.unimelb.edu.au/ snap/orb.html, 2005. 
[17] James Howie, A proof of the Scott-Wiegold conjecture on free products of cyclic groups, J. Pure Appl. Algebra 173 (2002), no. 2, 167-176, DOI 10.1016/S0022-4049(02)00042-7. MR.1915093 (2003h:20048)

[18] Kazuhiro Ichihara and Toshio Saito, Surgical distance between lens spaces, Tokyo J. Math. 34 (2011), no. 1, 153-164, DOI 10.3836/tjm/1313074448. MR2866640(2012h:57034)

[19] András Juhász, Holomorphic discs and sutured manifolds, Algebr. Geom. Topol. 6 (2006), 1429-1457, DOI 10.2140/agt.2006.6.1429. MR2253454(2007g:57024)

[20] P. Kutzko, On groups of finite weight, Proc. Amer. Math. Soc. 55 (1976), no. 2, 279-280. MR.0399272 (53 \#3123)

[21] W. B. R. Lickorish, A representation of orientable combinatorial 3-manifolds, Ann. of Math. (2) 76 (1962), 531-540. MR0151948 (27 \#1929)

[22] Marco Marengon, On d-invariants and generalised Kanenobu knots, arXiv preprint arXiv:1412.3433, 2014

[23] José María Montesinos, Classical tessellations and three-manifolds, Universitext, SpringerVerlag, Berlin, 1987. MR915761 (89d:57016)

[24] Louise Moser, Elementary surgery along a torus knot, Pacific J. Math. 38 (1971), 737-745. MR0383406 (52 \#4287)

[25] David Mullins, The generalized Casson invariant for 2-fold branched covers of $S^{3}$ and the Jones polynomial, Topology 32 (1993), no. 2, 419-438, DOI 10.1016/0040-9383(93)90029-U. MR $1217078(94 \mathrm{~h}: 57013)$

[26] Robert Myers, Excellent 1-manifolds in compact 3-manifolds, Topology Appl. 49 (1993), no. 2, 115-127, DOI 10.1016/0166-8641(93)90038-F. MR1206219 (94a:57034)

[27] Yi Ni, Link Floer homology detects the Thurston norm, Geom. Topol. 13 (2009), no. 5, 2991-3019, DOI 10.2140/gt.2009.13.2991. MR2546619(2010k:57032)

[28] Peter Ozsváth and Zoltán Szabó, Absolutely graded Floer homologies and intersection forms for four-manifolds with boundary, Adv. Math. 173 (2003), no. 2, 179-261, DOI 10.1016/S0001-8708(02)00030-0. MR1957829 (2003m:57066)

[29] Peter Ozsváth and Zoltán Szabó, Holomorphic disks and genus bounds, Geom. Topol. 8 (2004), 311-334, DOI 10.2140/gt.2004.8.311. MR2023281 (2004m:57024)

[30] Peter S. Ozsváth and Zoltán Szabó, Knot Floer homology and rational surgeries, Algebr. Geom. Topol. 11 (2011), no. 1, 1-68, DOI 10.2140/agt.2011.11.1. MR2764036(2012h:57056)

[31] Martin Scharlemann, Crossing changes, Chaos Solitons Fractals 9 (1998), no. 4-5, 693704, DOI 10.1016/S0960-0779(97)00105-7. Knot theory and its applications. MR.1628751 (99b:57022)

[32] Peter Scott, The geometries of 3-manifolds, Bull. London Math. Soc. 15 (1983), no. 5, 401487, DOI 10.1112/blms/15.5.401. MR705527 (84m:57009)

[33] William Thurston, The geometry and topology of 3-manifolds. Princeton University, Mimeographed lecture notes, 1977.

[34] William P. Thurston, Hyperbolic structures on 3-manifolds. I. Deformation of acylindrical manifolds, Ann. of Math. (2) 124 (1986), no. 2, 203-246, DOI 10.2307/1971277. MR855294 (88g:57014)

[35] Andrew H. Wallace, Modifications and cobounding manifolds, Canad. J. Math. 12 (1960), 503-528. MR0125588 (23 \#A2887)

School of Mathematics and Statistics, University of Melbourne, Parkville, ViC 3010, Australia

E-mail address: nhoffman@ms.unimelb.edu.au

Department of Mathematics, Bromfield-Pearson Hall, Medford, MA, USA

E-mail address: genevieve.walsh@tufts.edu 